\title{
Estimativa da Rotação em Motores de Indução Usando Histograma de Gradientes Orientados
}

\author{
Cleber Gustavo Dias* Kessia Lucas Rodrigues* \\ Luiz Carlos da Silva* Clayton Costa Sakamae** \\ Thiago Dorgam Marcassa ${ }^{* *}$ Thales Santos Ferreira ${ }^{* *}$ \\ * Programa de Mestrado e Doutorado em Informática e Gestão do \\ Conhecimento,UNINOVE, SP, (e-mails: diascg@uni9.pro.br, \\ kessiakk.ppgi@gmail.com, luiz.carlos.silva@uni9.pro.br). \\ ** Bacharelado em Engenharia Elétrica, UNINOVE, SP, (e-mails: \\ claytonsakamae@gmail.com,tdmarcassa@gmail.com, \\ thsantferr@gmail.com)
}

\begin{abstract}
This paper presents the use of histogram of oriented gradients technique, usually applied in the image processing field, to estimate the slip in squirrel cage induction motors. Such value is achieved by monitoring only one machine phase current with sample time in $250 \mathrm{~ms}$, wich allows future real time applications. The features extracted with the histograms were used as inputs for a neural network to estimate the final rotation of the machine. The results obtained from some experimental tests are presented to validate the present approach.

Resumo: O presente artigo aborda o uso da técnica de histograma de gradientes orientados, normalmente aplicada na área de processamento de imagens, na estimativa da rotação em motores de indução trifásicos com rotor gaiola de esquilo. Tal grandeza é alcançada a partir do monitoramento de apenas uma das correntes de fase da máquina, empregando janelas amostrais de apenas $250 \mathrm{~ms}$, o que corrobora para futuras aplicações em tempo real. As características extraídas com o apoio dos histogramas foram aplicadas em uma rede neural para a estimativa final da rotação de eixo da máquina. Os resultados obtidos a partir de alguns ensaios experimentais são apresentados no sentido de validar a abordagem proposta.
\end{abstract}

Keywords: induction motor; rotation speed, oriented gradient histogram, load torque, condition monitoring, rotation estimation.

Palavras-chaves: motor de indução, velocidade de rotação, histograma de gradientes orientados, torque de carga, monitoramento das condições, estimativa de rotação.

\section{INTRODUÇÃO}

A informação da velocidade rotórica de um motor de indução trifásico (MIT) é extremamente relevante para os mais diversos tipos de aplicações industriais, tais como nos controles de velocidade, posição, torque (Merabet et al. (2017)), na estimativa do rendimento da máquina, ou ainda na deteç̧ão de eventuais falhas que podem comprometer a sua estrutura (Roque (2015)).

O trabalho publicado por Lu et al. (2006), por exemplo, realizou uma importante revisão da literatura e descreveu os principais métodos voltados para a avaliação de perdas e da eficiência nos motores de indução trifásicos conhecidos até aquele momento, sendo muitos deles empregados e/ou estudados até os dias de hoje. Neste estudo fica evidente que a maioria dos métodos necessitam da informação da velocidade de eixo do motor para se conhecer a sua efici-

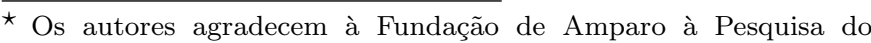
Estado de São Paulo - FAPESP pelo suporte financeiro (processo $\left.n^{\circ} 2018 / 05214-2\right)$
}

ência.

O artigo de Silva et al. (2015a) propôs um método voltado para a estimativa do torque de carga em motores de indução, sem o uso de qualquer acoplamento mecânico. Neste caso, foi necessário empregar a chamada Transformada Z Chirp em tempo curto (short time chirp z transform - sigla STCZT em inglês), a fim de estimar com precisão o escorregamento do eixo, além de ser necessário o conhecimento do número de barras do rotor para avaliar o espectro de frequências e as harmônicas de interesse.

Como mencionado, outros estudos corroboram com a necessidade de se conhecer a rotação da máquina, a fim de extrair informações relevantes para se detectar eventuais avarias no MIT, quer seja no seu estator, ou mesmo na gaiola rotórica (Antonino-Daviu et al. (2016) e Naha et al. (2016)).

De modo geral, a rotação do eixo pode ser medida com o uso de um encoder, ou mesmo um tacômetro ótico, mas o primeiro exige um acoplamento mecânico entre o rotor e o instrumento de medição, além de uma calibração ex- 
tremamente cuidadosa, e o segundo o uso de fitas adesivas refletivas, no sentido de aferir a velocidade do eixo. De todo o modo, em um ambiente industrial muitas vezes não é possível usar este método de medição, devido à falta de iluminação do local, as impurezas ou mesmo ao difícil acesso por parte de um profissional da área de manutenção Ferreira and Lopes (2016).

Neste sentido, nos últimos anos muitos trabalhos propuseram técnicas capazes de estimar a rotação de eixo do motor e o seu escorregamento, a fim de evitar o acoplamento de sensores invasivos no MIT, ou ainda o uso dos tacômetros, durante a operação das máquinas em ambiente fabril.

O estudo publicado por Dongfeng et al. (2006) apresentou uma abordagem orientada para a estimativa da velocidade de eixo de um MIT, usando as transformadas de Hilbert e interpolada de Fourier. Embora este trabalho tenha alcançado um erro bastante reduzido para a rotação estimada, frente aos resultados experimentais, o modelo proposto necessita de duas técnicas de processamento de sinais e sugere a sua implementação em dispositivos do tipo DSP, normalmente de custo relativamente elevado quando se trata de motores de indução de baixa potência, por exemplo.

O trabalho apresentado por Orman et al. (2011) descreve as dificuldades em se determinar o escorregamento do motor em baixa rotação e sugere um método fundamentado na transformada rápida de Fourier, de modo a estimar a velocidade de eixo naquela condição operacional. Todavia, esta abordagem necessita de uma alta resolução em frequência para estimar as harmônicas de interesse, além do fato de estar muitas vezes sujeita a harmônicas indesejáveis quando o MIT é alimentado por um inversor, por exemplo.

O método proposto por Silva et al. (2015b) oferece uma abordagem bastante interessante para a estimativa da rotação e do escorregamento da máquina, na condição de cargas oscilatórias. Trata-se de um método aplicado no domínio da frequência, ou seja, torna-se necessário encontrar as frequências harmônicas de interesse para estimar o escorregamento, além do fato de necessitar dos dados construtivos do rotor para a sua aplicação.

Tomando por base os trabalhos acima descritos, a presente pesquisa propõe uma abordagem alternativa, no domínio do tempo, usando histogramas de gradientes orientados, como será melhor descrito na próxima seção, para estimar a rotação de eixo de um motor de indução trifásico, o mesmo foi alimentado por um inversor de frequência, as caraterísticas foram extraídas de uma das fases da máquina, coletadas por meio de um transformador de corrente (TC), foi mencionado que muitas vezes o ambiente fabril dificulta a medição de rotação por meio de tacômetro ou encoder, o uso de um TC acaba sendo simples, porque se trata de um sensor não invasivo que é acoplado na fase de alimentação do motor de indução trifásico.

\section{O USO DE HISTOGRAMAS DE GRADIENTES ORIENTADOS NA ESTIMATIVA DA ROTAÇÃO}

Nesta seção são apresentados os fundamentos relacionados ao Histograma de Gradientes Orientados (HGO), bem como a sua aplicação na extração de características da corrente de fase, a fim de estimar a rotação no eixo de um MIT.

\subsection{Fundamentos do $H G O$}

Os Histogramas de Gradientes Orientados (HGO) foram originalmente criados com a finalidade de extrair características de um objeto presente em uma imagem, mais particularmente para detectar humanos em uma imagem, como descrito nos trabalhos publicados por Dalal and Triggs (2005) e Tan et al. (2014). Mais recentemente, os HGO foram aplicados na detecção do rompimento de barras rotóricas em um motor de indução trifásico (Silva et al. (2018)).

De forma mais detalhada, a determinação de um HGO implica no cálculo das derivadas de um sinal discretizado, a fim de se obter o módulo de uma determinada amostra e o ângulo formado entre as suas amostras vizinhas. Quando se considera o pixel de uma imagem, por exemplo, a amplitude deste chamado pixel alvo em relação aos seus pixels vizinhos, bem como a orientação em relação a tais valores, são calculados pelas equações 1 e 2 a seguir:

$$
\begin{gathered}
z(x, y)=\sqrt{(I(x+1, y)-I(x-1, y))^{2}+(I(x, y+1)-I(x, y-1))^{2}} \\
\theta(x, y)=\arctan \left(\frac{I(x, y+1)-I(x, y-1)}{I(x+1, y)-I(x-1, y)}\right)
\end{gathered}
$$

Logo, a equação 1 refere-se ao cálculo do módulo e a equação 2 à orientação do gradiente obtido. A seguir é descrita a aplicação do HGO na estimativa da rotação de eixo de um motor de indução trifásico.

\subsection{Cálculo do HGO para a Extração de Características da Corrente}

Como descrito na seção anterior, o HGO foi originalmente proposto para encontrar objetos em uma dada imagem, e os seus fundamentos foram aqui aplicados para avaliar o escorregamento de um motor de indução trifásico. Para tal finalidade, as equações 1 e 2 foram modificadas em um sinal senoidal discretizado, ou seja, na corrente de fase do estator amostrada.

Deste modo, para uma dada amostra $A_{v}(x, y)$ selecionada de um sinal senoidal discretizado, foi determinado um triângulo formado pelas amostras imediatamente anterior e superior. Assim, foi calculado o módulo, ou amplitude, do triângulo e o seu ângulo de inclinação. Tais grandezas correspondem ao módulo e a orientação do gradiente, uma vez que as derivadas consideradas são as diferenças obtidas nos eixos x e y, como descrito nas equações 3 e 4 a seguir:

$$
\begin{aligned}
& z\left(A_{v}(x, y)\right)=\sqrt{(\Delta x)^{2}+(\Delta y)^{2}} \\
& \theta\left(A_{v}(x, y)\right)=\arctan \left(\frac{\Delta y}{\Delta x}\right) \\
& \text { Onde: } \Delta x=x_{n_{2}}-x_{n_{1}} \\
& \Delta y=y_{n_{2}}-y_{n_{1}}
\end{aligned}
$$

Os pontos $\left(x_{n_{1}}, y_{n_{1}}\right)$ e $\left(x_{n_{2}}, y_{n_{2}}\right)$ correspondem as amostras inferior e superior respectivamente a amostra $A_{v}(x, y)$. O histograma final para cada janela amostral do sinal de corrente é calculado a partir do módulo e ângulo obtidos de cada amostra e a distribuição das grandezas calculadas 
depende da distribuição dos chamados "bins", ou intervalos. Assim, dependendo do ângulo calculado para cada amostra o seu valor é ponderado para uma faixa superior, caso esteja mais próxima, ou para uma faixa inferior no caso contrário $\left(\omega_{k}(x, y)\right)$.

O histograma final, considerando o intervalo de ângulos, ou bins, foi calculado para uma janela amostral $W_{i}$ da corrente de fase, usando a equação 5 :

$$
\left[\operatorname{HGO}\left(u, W_{i}\right)\right](k)=\sum_{(x, y) \in W_{i}} \omega_{k}(x, y) z(x, y),
$$

Para $k=1,2, \ldots, n_{\text {bins }}$.

Onde: $u=$ sinal de corrente de fase do estator

\subsection{Aplicação de uma Rede Neural na Estimativa da Rotação usando $\mathrm{HGO}$}

Após a criação dos histogramas para cada janela amostral de corrente uma base de dados de treinamento e outra de validação foram geradas, a fim de estimar a rotação de eixo da máquina usando uma rede neural do tipo Perceptron Multicamadas. A Figura 1 ilustra a estrutura da rede neural modelada no presente trabalho.

As entradas são formadas pelas amplitudes de cada ângulo gerado após o cálculo do HGO e a saída compreende a rotação da máquina estimada.

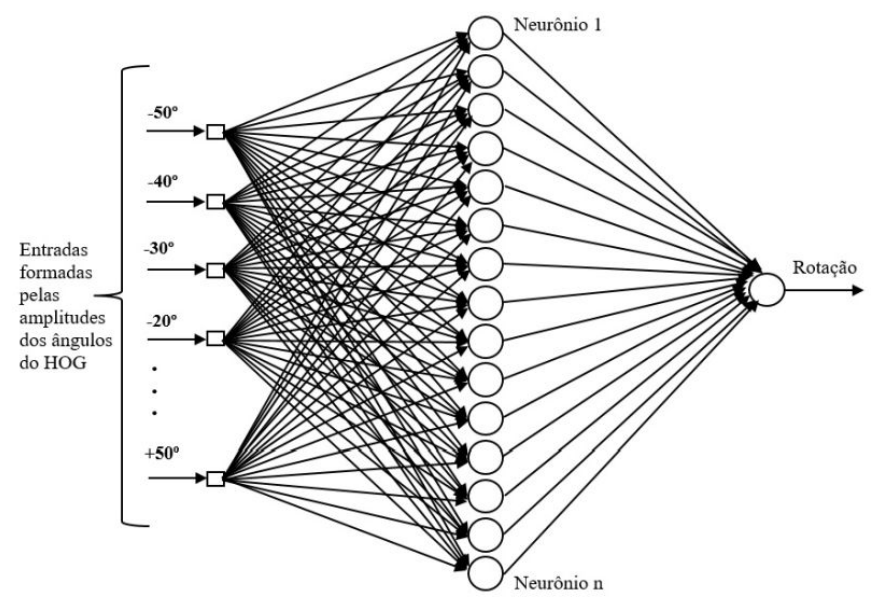

Figura 1. Estrutura da rede neural modelada com dados do HGO

A rede neural foi treinada usando o algoritmo LevenbergMarquardt (LM) e o seu desempenho foi avaliado usando o erro absoluto médio (EAM), conforme a equação 6 . Como descrito por Hagan and Menhaj (1994), embora o algoritmo LM apresente um custo computacional maior para o treinamento, a sua aplicação é justificada pelo desempenho obtido, além de ser bastante eficiente para uma rede com algumas centenas de pesos sinápticos, razão pela qual tal método foi escolhido para este trabalho.

$$
E A M=\frac{1}{N} \sum_{n=1}^{N}\left|y_{\text {medido }}(n)-y_{\text {estimado }}(n)\right|
$$

Onde:

$y_{\text {medido }}(n)=$ saída medida, ou seja, rotação do eixo medida com um tacômetro;

yestimado $(n)=$ saída estimada pela rede neural (rotação estimada)

\section{APARATO EXPERIMENTAL E RESULTADOS OBTIDOS}

\subsection{Estrutura do Aparato Montado em Laboratório}

A seguir é apresentado todo o aparato experimental montado para avaliar a rotação de eixo do motor de indução, para posterior estimação usando a abordagem ora proposta. As principais partes voltadas para a coleta dos dados são apresentados na Figura 2. Para tal configuração, foram realizados um total de 140 experimentos, a fim de aferir a velocidade de eixo do motor a partir de uma condição praticamente a vazio, até uma operação próxima a carga nominal da máquina.

O motor ensaiado em laboratório tem uma potência nominal de $7,5 \mathrm{~kW}$, alimentação em $60 \mathrm{~Hz}, 4$ pólos, e escorregamento nominal próximo a 3,33\% (1740 rpm). A máquina está acoplada a um freio eletromagnético do tipo Foucault, capaz de aplicar diferentes cargas ao seu eixo.

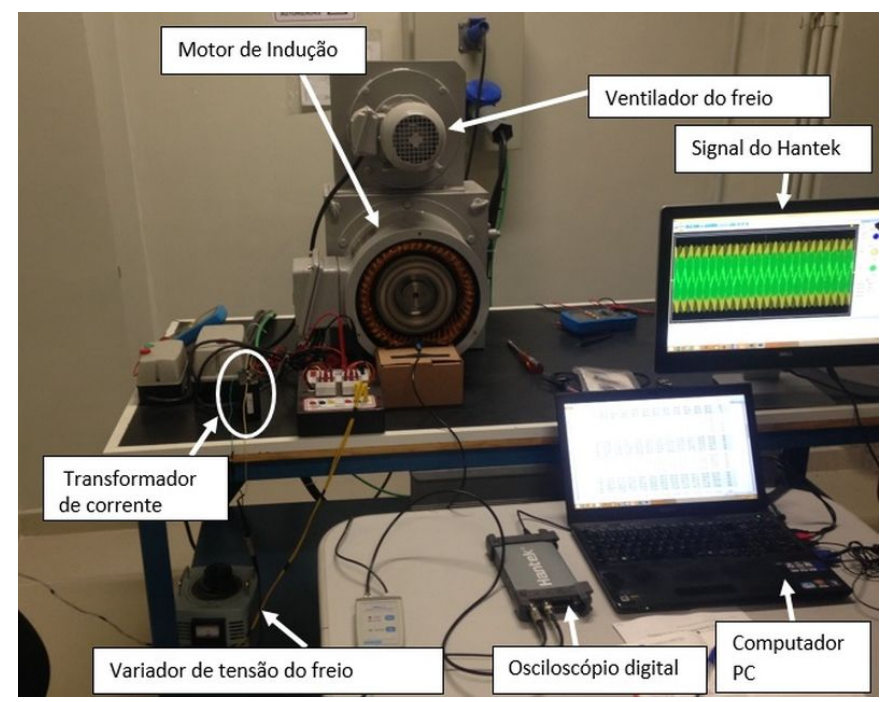

Figura 2. Aparato experimental montado em laboratório

Os experimentos foram conduzidos no sentido de amostrar a corrente de uma das fases do motor em uma janela de 10s, para uma frequência de amostragem igual a $10 \mathrm{kHz}$. Para cada condição de carga do motor, foram coletadas 10 amostras da corrente do estator. O sinal foi medido com um transformador de corrente (TC), na relação de $20 \mathrm{~A} / 1 \mathrm{~V}$, e uma placa de aquisição de dados da National Instruments, modelo USB-6003, foi empregada para amostrar o sinal e enviá-lo para um computador do tipo PC (processador Intel i7, memória de $16 \mathrm{~GB}$ ). Os dados também foram amostrados em um osciloscópio digital do tipo Hantek, conectado em uma das portas USB do computador.

Vale mencionar ainda que, os dados foram suavizados com um filtro passa-baixas do tipo Butterworth de $6^{\text {a }}$ ordem, em uma frequência de corte de $200 \mathrm{~Hz}$, a fim de evitar maiores distorções no sinal de corrente devido à presença de componentes harmônicas indesejadas. O motor foi acionado por um inversor de frequência WEG CFW-08, configurado no modo escalar (V/Hz). Os dados 
obtidos pela rede neural foram comparados com os dados medidos por meio de um tacômetro ótico da marca Minipa e modelo MDT-2238.

O filtro passa-baixas, a segmentação do sinal de corrente e a montagem dos histogramas foram realizados usando o software Phyton e a rede neural foi montada e testada com o apoio do software Matlab.

\subsection{Resultados Experimentais}

Conforme descrito anteriormente, o motor foi acionado para diferentes condições de carga, em um total de 140 experimentos (10 amostras de corrente em cada caso), conforme mostrado na Tabela 1. Após a coleta do sinal de corrente em janelas de 10s, cada amostra foi segmentada em janelas menores de $0,25 \mathrm{~s}(250 \mathrm{~ms})$, com o objetivo de construir uma base de dados representativa em um domínio de tempo capaz de promover a estimativa da rotação do motor em tempo real.

Deste modo, foi criado um histograma para cada janela de $250 \mathrm{~ms}$ e uma base de dados de treinamento e outra de validação foram montadas para aplicação na rede neural artificial. A base de treinamento foi construída com 1160 amostras, incluindo os ensaios 1,2,3,4,5,6,8,10,11,13 e 14 . A base de validação foi construída com 240 amostras, incluindo os ensaios 7 (1776 rpm), 9 (1768 rpm) e 12 (1752 rpm). O fato dos ensaios citados na validação não estarem presentes nos ensaios de treinamento foi muito importante para evitarmos o problema de generalização dos dados, circunstância que tornaria o modelo em treinamento não muito confiável.

Tabela 1. Tipos de experimentos realizados com o motor de indução

\begin{tabular}{lccc}
\hline Ensaio & Vfreio & Rotacao $($ rpm $)$ & $s(\%)$ \\
\hline 1 & $10 \mathrm{~V}$ & 1796 & 0,22 \\
2 & $20 \mathrm{~V}$ & 1795 & 0,28 \\
3 & $30 \mathrm{~V}$ & 1793 & 0,39 \\
4 & $40 \mathrm{~V}$ & 1788 & 0,66 \\
5 & $50 \mathrm{~V}$ & 1785 & 0,83 \\
6 & $60 \mathrm{~V}$ & 1782 & 1 \\
7 & $70 \mathrm{~V}$ & 1776 & 1,33 \\
8 & $80 \mathrm{~V}$ & 1771 & 1,61 \\
9 & $90 \mathrm{~V}$ & 1768 & 1,77 \\
10 & $100 \mathrm{~V}$ & 1760 & 2,22 \\
11 & $110 \mathrm{~V}$ & 1758 & 2,38 \\
12 & $120 \mathrm{~V}$ & 1752 & 2,67 \\
13 & $130 \mathrm{~V}$ & 1746 & 3 \\
14 & $140 \mathrm{~V}$ & 1743 & 3,16 \\
\hline
\end{tabular}

Para o cáculo dos histogramas foi avaliada uma distribuição dos ângulos em intervalos de $10^{\circ}$ e como os ângulos inferiores a $-50^{\circ}$ e superiores a $+50^{\circ}$ apresentaram uma amplitude igual a zero, foram consideradas entradas válidas para o modelo neural somente com as amplitudes dos ângulos que variaram na faixa de $-50^{\circ}$ e $+50^{\circ}$, conforme ilustrado na Figura 3.

Na Figura 3 é possível notar que as amplitudes variam para cada ângulo do histograma em função da carga aplicada ao eixo do motor, logo tal característica foi fundamental para se estimar o escorregamento da máquina. Em geral, a medida que a carga é incrementada a amplitude do respectivo ângulo do histograma é reduzida, além do fato de que os ângulos das extremidades são preenchidos a

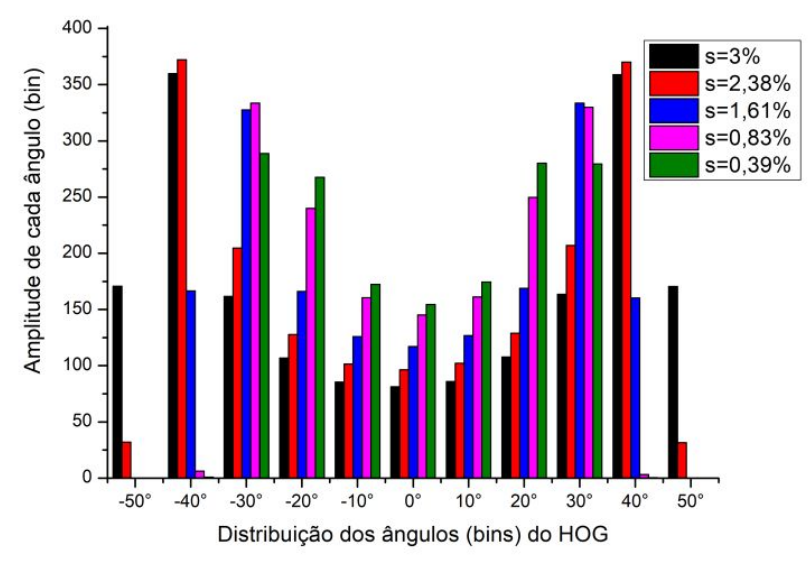

Figura 3. Variação de amplitude dos histogramas em função da carga (escorregamento)

medida que a carga é elevada. Assim, com a distribuição proposta, a rede neural foi concebida com 11 entradas para a faixa de ângulos proposta e uma única saída.

A rede neural foi treinada com uma única camada escondida, no caso com 93 neurônios, usando a função de ativação tangente hiperbólica. A Figura 4 ilustra a saída estimada pela rede neural para uma carga constante no eixo do motor, em uma rotação medida de 1776 rpm. Notase que a rede foi capaz de estimar tal condição de operação da máquina, uma vez que o seu valor médio oscilou em torno da rotação de referência.

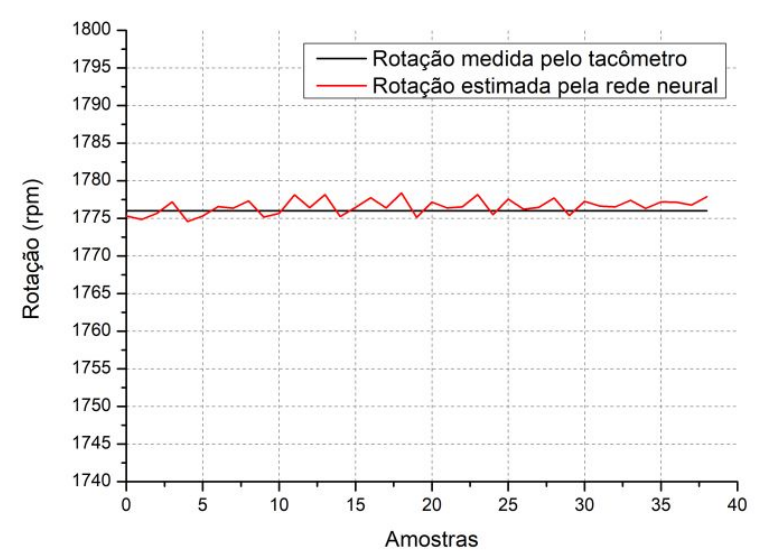

Figura 4. Comparação entre os valores estimados e medidos para uma rotação em 1776 rpm

A Figura 5 mostra a saída estimada para uma outra condição de carga do motor, para uma rotação de 1768 rpm. A rede foi capaz de estimar com boa acuidade tal valor, embora tenha apresentado uma tendência em estimar uma velocidade um pouco superior àquela de referência.

Por sua vez, a Figura 6 mostra os resultados obtidos para uma condição de carga mais próxima a nominal da máquina, e nesta rotação a rede apresentou uma oscilação maior, muito embora tenha sugerido uma estimativa de 


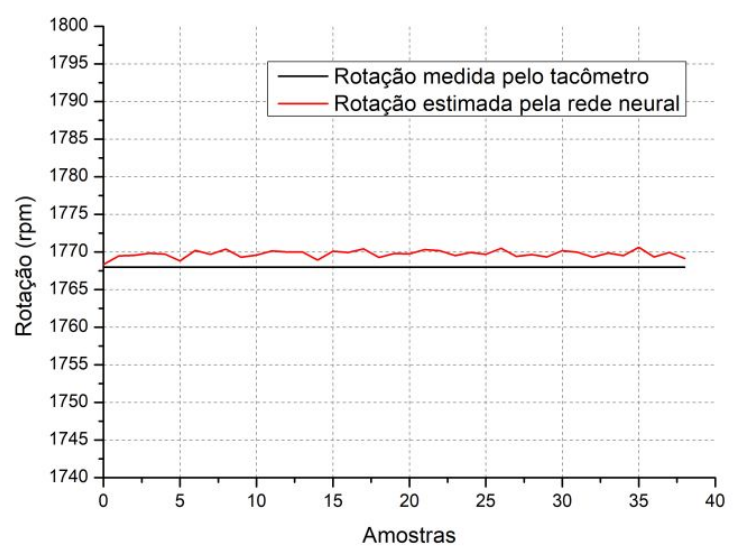

Figura 5. Comparação entre os valores estimados e medidos para uma rotação em $1768 \mathrm{rpm}$

velocidade com tendência a uma aproximação ao valor de referência em algumas amostras.

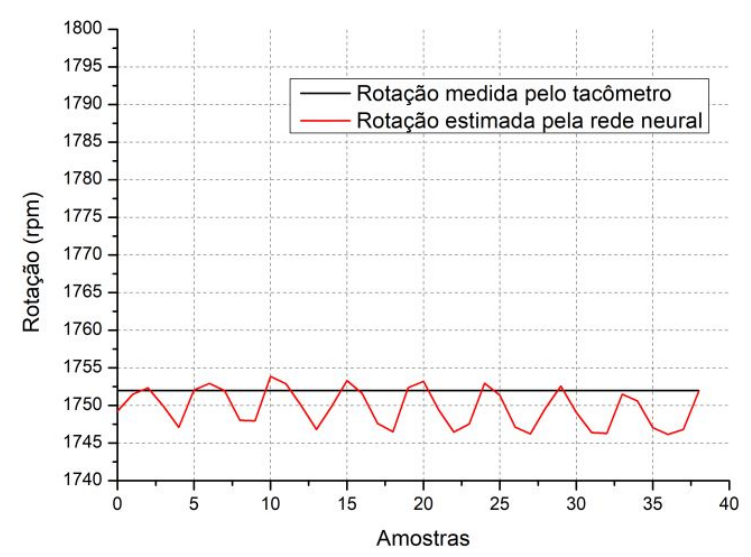

Figura 6. Comparação entre os valores estimados e medidos para uma rotação em $1752 \mathrm{rpm}$

Os resultados mostrados na Tabela 2 mostram uma boa aproximação entre os resultados estimados pela rede neural e aqueles medidos com o tacômetro. De modo geral, nota-se que há um erro maior para uma condição de carga maior no eixo da máquina, ou seja, para um escorregamento maior. Neste caso, novos estudos devem ser realizados no sentido de melhorar esta estimativa para o motor com uma carga maior, provavelmente incrementando os ensaios nesta condição para o treinamento da rede neural. Embora o erro médio observado nas 40 amostras tenha alcançado um valor abaixo de $1 \%$, por exemplo, ainda é necessário avaliar os intervalos de ângulos do HGO e incrementar o treinamento da rede neural no sentido de reduzir o EAM em cada caso.

\section{CONCLUSÃO}

Este trabalho apresentou uma abordagem alternativa para a estimativa da rotação de eixo de um motor de indução
Tabela 2. Valores de erros obtidos em cada velocidade de eixo

\begin{tabular}{lccc}
\hline Rotação (rpm) & EAM (rpm) & Erro médio (\%) & Desvio Padrao \\
\hline 1776 & 3,50 & 0,19 & 2,62 \\
1768 & 2,28 & 0,13 & 1,26 \\
1752 & 4,39 & 0,25 & 2,77 \\
\hline
\end{tabular}

trifásico, usando apenas características extraídas da corrente de fase da máquina, por meio de um transformador de corrente (TC). Para tal finalidade, foi calculado o histograma de gradientes orientados, normalmente aplicado na área de processamento de imagens, de forma a obter os ângulos e as suas amplitudes mais representativas para cada condição de carga do motor.

Uma rede neural artificial foi utilizada como modelo de regressão capaz de estimar a velocidade de eixo da máquina, a partir das grandezas extraídas com o suporte do HGO. O resultado da rede foi comparado com aquele medido com um tacômetro óptico.

Os resultados obtidos mostraram uma boa aproximação entre a rotação medida e aquela estimada pela rede neural em três condições operacionais de carga. Todavia, novas investigações devem ser realizadas no sentido de avaliar o uso do HGO com o motor operando em carga variável em seu eixo. Ademais, devem ser ainda pesquisadas as melhores configurações do histograma, ou seja, a distribuição de intervalos dos ângulos, a fim de melhorar as estimativas obtidas não apenas para a carga constante, mas também para cargas oscilatórias, além de investigar a melhor frequência de amostragem e de corte para o filtro passa baixas.

De todo o modo, o uso dos histogramas de gradientes orientados surge como uma abordagem promissora para o problema da estimativa da rotação de eixo em um motor de indução trifásico, o que corrobora para a sua aplicação mais direta em um ambiente industrial, dada a facilidade de aquisição da corrente de fase e a disponibilidade do calculado simplificado dos histogramas em um hardware dedicado.

\section{AGRADECIMENTOS}

Os autores agradecem à FAPESP pelo apoio financeiro (processo $\mathrm{n}^{\circ} 2018 / 05214-2$ ) e a Universidade Nove de Julho (UNINOVE) por todo o suporte oferecido na montagem do laboratório e apoio técnico para a condução dos experimentos.

\section{REFERENCIAS}

Antonino-Daviu, J.A., Pons-Llinares, J., and Lee, S.B. (2016). Advanced rotor fault diagnosis for mediumvoltage induction motors via continuous transforms. IEEE Transactions on Industry Applications, 52(5), 4503-4509.

Dalal, N. and Triggs, B. (2005). Histograms of oriented gradients for human detection. In 2005 IEEE Computer Society Conference on Computer Vision and Pattern Recognition (CVPR'05), volume 1, 886-893 vol. 1.

Dongfeng, D., Unsworth, P.J., and Gao, R.X. (2006). Sensorless speed measurement of induction motor using hilbert transform and interpolated fast fourier transform. IEEE Transactions on Instrumentation and Measurement, 55(1), 290-299. 
Ferreira, F.J.T.E. and Lopes, F.J.P. (2016). Webcambased tachometer for in-field induction motor load estimation. In 2016 XXII International Conference on Electrical Machines (ICEM), 2380-2388.

Hagan, M.T. and Menhaj, M.B. (1994). Training feedforward networks with the marquardt algorithm. IEEE Transactions on Neural Networks, 5(6), 989-993.

Lu, B., Habetler, T.G., and Harley, R.G. (2006). A survey of efficiency-estimation methods for in-service induction motors. IEEE Transactions on Industry Applications, 42(4), 924-933.

Merabet, A., Tanvir, A.A., and Beddek, K. (2017). Torque and state estimation for real-time implementation of multivariable control in sensorless induction motor drives. IET Electric Power Applications, 11(4), 653663.

Naha, A., Samanta, A.K., Routray, A., and Deb, A.K. (2016). A method for detecting half-broken rotor bar in lightly loaded induction motors using current. IEEE Transactions on Instrumentation and Measurement, 65(7), 1614-1625.

Orman, M., Orkisz, M., and Pinto, C.T. (2011). Slip estimation of a large induction machine based on mcsa. In 8th IEEE Symposium on Diagnostics for Electrical Machines, Power Electronics Drives, 568-572.

Roque, L. (2015). Estimação da velocidade do motor de indução através do algoritmo de aproximação senoidal na corrente do estator. Dissertação de Mestrado, Universidade Federal de Itajubá, p.108.

Silva, L., C.G, D., and Alves, W.A. (2018). A histogram of oriented gradients for broken bars diagnosis in squirrel cage induction motors. Lecture Notes in Computer Science, 11139, 33-42.

Silva, W.L., Lima, A.M.N., and Oliveira, A. (2015a). A method for measuring torque of squirrel-cage induction motors without any mechanical sensor. IEEE Transactions on Instrumentation and Measurement, 64(5), 12231231.

Silva, W.L., Lima, A.M.N., and Oliveira, A. (2015b). Speed estimation of an induction motor operating in the nonstationary mode by using rotor slot harmonics. IEEE Transactions on Instrumentation and Measurement, 64(4), 984-994.

Tan, H., Yang, B., and Ma, Z. (2014). Face recognition based on the fusion of global and local hog features of face images. IET Computer Vision, 8(3), 224-234. 